\title{
CALCULATION OF EFFECTIVE ELASTIC MODULI OF TEXTURED MATERIALS
}

\author{
N. J. PARK ${ }^{1}$, H. J. BUNGE ${ }^{1}$, H. KIEWEL ${ }^{2}$ and L. FRITSCHE ${ }^{2}$ \\ ${ }^{\prime}$ Department of Physical Metallurgy \\ ${ }^{2}$ Institute of Theoretical Physics B, Technical University Clausthal, Germany
}

(Received 12 September 1994)

Effective elastic constants of polycrystalline materials were determined with a recently developed method. This method bases on the modelation of the actual material by a cluster of 100 to 500 single crystals. In the present version of the scheme parallelepipeds are used. The ODF was calculated with the series expansion method. The transformation of this ODF into a finite sum of single orientations permits to assign any grain an individual orientation.

Reliable results for the effective elastic moduli of textured materials are reported. They lie always within the bounds of Voigt and Reuss. The very high anisotropic substances, e.g. shape-memory-alloys, show a significant deviation from the Hill values.

KEY WORDS: Elastic moduli, effective elastic constants, ODF-calculation and ODF-simulation.

\section{INTRODUCTION}

First attempts to gain access to the effective elastic moduli of polycrystalline substances were carried out by Voigt (1910) and Reuss (1929). These methods base on the assumption that throughout the whole material the strain or the stress is constant. For that reason the moduli of Voigt and Reuss are simply volume averages of the orientation-dependent stiffnesses and compliances, respectively. As a consequence the effect of the grain interaction is neglected. Hill (1952) shows that the actual effective constants lie within these values of Voigt and Reuss. For many samples the arithmetic mean of these two values the so-called Hill value approximates the experimental data quite well. For highly anisotropic materials the Hill value fails. An overview of these and some other conservative methods has recently been published (Morawiec, 1994).

The first successful method which takes the grain interaction into consideration was developed by Kröner (Kröner, 1958; Kneer, 1964; Kneer, 1965; Morris, 1971). If there is no correlation between the grains and the mean grain shape is spherical this method yields the exact polycrystal constants (Kröner, 1977).

The vastly increasing capacity of modern electronic computers in the past decade leads to new approaches in material sciences. The recently developed schemes by Kumar (1992) and Kiewel and Fritsche (1994a, 1994b) base on the modelation of the actual material by an aggregate of 100-1000 grains. This aggregate is subjected to an homogeneous deformation at the entire surface of the cluster. The stress and strain field inside the cluster are then calculated. The stress-strain relation of the macroscopic homogeneous sample yields its effective elastic moduli. 


\section{METHOD}

The texture of a polycrystalline material is defined by the orientation distribution function (ODF) of its crystallites. Diffraction methods such as X-ray diffraction allow only the determination of pole density distribution functions, the so-called pole figures. They are two-dimensional projections of the three-dimensional ODF. Hence, pole figures are not a unique representation of the texture. This is more serious the more complicated the texture is. The ODF can, however, be calculated mathematically from several pole figures. This has been called pole figure inversion or ODF-analysis. The most frequently used method.bases on the expansion of the ODF in generalised spherical harmonics (Bunge, 1982).

After determining the ODF with this series expansion method we transform it into a finite sum of single orientations. For that purpose we calculate the values of the ODF on a grid in the Euler space. Values which are less than zero are replaced by zero since the ODF is a density and therefore non-negative. We integrate the ODF over a narrow region of the Euler space. If the integrated value is greater than one we identify this region with a single orientation. Varying the size of the integration region guarantees that the whole ODF is transformed into a sum of single orientations. The number of these orientations is variable since the ODF is determined apart from a normalisation constant.

After explaining the determination of the individual orientations of the grains we give a brief description of the recently developed method to calculate effective elastic constants (for details see (Kiewel and Fritsche, 1994b)). We confine ourselves to purely static deformations because we are only interested in the calculation of effective moduli. The strain tensor $\underline{\underline{\sigma}}(\underline{r})$ fulfils the relation

$$
\underline{\nabla} \cdot \underline{\underline{\sigma}}(\underline{r})=\underline{0}
$$

in the absence of external forces. In restriction on linear elasticity theory the strain tensor is defined by

$$
\varepsilon_{i j}(\underline{r}) \stackrel{\text { def }}{=} \frac{1}{2}\left(\frac{\partial u_{i}(\underline{r})}{\partial x_{j}}+\frac{\partial u_{j}(\underline{r})}{\partial x_{i}}\right) .
$$

The displacement is denoted by $\underline{u}(\underline{r})$. The stress-strain relation is identical with the generalised Hooke's law:

$$
\sigma_{i j}(\underline{r})=\sum_{k, l} c_{i j k l}^{(\alpha)} \varepsilon_{k l}(\underline{r}),
$$

where $\alpha$ numbers the grains. In combining these three Eqs. (1), (2) and (3) we arrive at the fundamental differential equation:

$$
\sum_{j, k, l} c_{i j k l}^{(\alpha)} \frac{\partial^{2}}{\partial x_{j} \partial x_{l}} u_{k}^{(\alpha)}(\underline{r})=0 \quad \text { for all } \quad i
$$

In the present work the Dirichlet problem is solved, i.e. we look for the solution of (4) under the constraint

$$
\left.\underline{u}\right|_{S}(\underline{x})=\underline{u}_{0}(\underline{x})
$$

at the entire surface $S$ of the cluster. The given displacement $\underline{u}_{0}(\underline{x})$ is chosen that it results in a constant strain $\underline{\underline{\varepsilon}}_{0}$. 
We expand $\underline{u}^{(\alpha)}(\underline{r})$ in each grain in terms of basis functions $\underline{u}_{n}^{(\alpha)}(\underline{r})$ which satisfy individually Eq. (4):

$$
\underline{u}^{(\alpha)}(\underline{r})=\sum_{n} a_{n}^{(\alpha)} \underline{u}_{n}^{(\alpha)}(\underline{r})
$$

where $a_{n}^{(\alpha)}$ denotes the expansion coefficients. (For the construction of these basis functions see (Kiewel and Fritsche, 1994b).) At the grain boundaries $S_{\alpha, \alpha}$ the displacement and the stress $\underline{\sigma}_{\underline{n}}$ must be continuous, that is

and

$$
\left.\underline{u}^{\left(\alpha^{\prime}\right)}(\underline{r})\right|_{s_{\alpha, \alpha}}=\left.\underline{u}^{(\alpha)}(\underline{r})\right|_{s_{\alpha, \alpha}}
$$

$$
\left.{\underline{\sigma_{n}}}^{\left(\alpha^{\prime}\right)}(\underline{r})\right|_{s_{\alpha^{\prime}, \alpha}}=\left.\underline{\sigma}_{\underline{n}}^{(\alpha)}(\underline{r})\right|_{s_{\alpha, \alpha}}
$$

In general, these boundary conditions cannot be satisfied exactly. We relax these conditions by the requirement that the truncation error

$$
\begin{aligned}
E \stackrel{\text { def }}{=} \sum_{\alpha^{\prime}, \alpha} \int_{S_{\alpha^{\prime}, \alpha}}\left(\left|\underline{u}^{(\alpha)}(\underline{r})-\underline{u}^{(\alpha)}(\underline{r})\right|^{2}\right. & \\
& \left.+\lambda\left|\underline{\sigma}_{\underline{n}}^{(\alpha)}(\underline{r})-\underline{\sigma}_{\underline{n}}^{(\alpha)}(\underline{r})\right|^{2}\right) d S
\end{aligned}
$$

attain a minimum. The weight factor $\lambda$ guarantees that the two portions of the integrand contribute with approximately the same magnitude to the mismatch. On inserting Eqs. (6) and (5) in (9) and minimising this error we obtain a set of inhomogeneous linear equations which we cast as

$$
\underline{\underline{M}} \underline{a}=\underline{I} \text {. }
$$

The displacement $\underline{u}_{0}(\underline{x})$ at the surface of the cluster causes the inhomogeneity $\underline{I}$.

The solution vector $\underline{a}$ of (10) yields the displacement and therefore the strain and stress tensor inside the cluster. We form the averages of $\underline{\underline{\varepsilon}}(\underline{r})$ and $\underline{\underline{\sigma}}(\underline{r})$ over the volume of the cluster:

$$
\begin{aligned}
& \left(\overline{\varepsilon_{i j}}\right) \stackrel{\text { def }}{=} \frac{1}{V} \int_{V}\left(\varepsilon_{i j}(\underline{r})\right) d V, \\
& \left(\overline{\sigma_{i j}}\right) \stackrel{\text { def }}{=} \frac{1}{V} \int_{V}\left(\sigma_{i j}(\underline{r})\right) d V .
\end{aligned}
$$

The stress-strain relation of the macroscopic homogeneous material yields then the effective elastic constants $\hat{C}_{i j k l}$ :

$$
\overline{\sigma_{i j}}=\sum_{k, l} \hat{C}_{i j k l} \overline{\varepsilon_{k l}} \text {. }
$$

\section{RESULTS AND DISCUSSION}

\section{Test of the $O D F$-simulation}

We first test our scheme transforming an ODF into a finite sum of single orientations. For that purpose we compare results obtained with the ODF-simulation scheme with 
that directly calculated from the coefficients $C_{i}^{\mu \nu}$ of the series expansion method described in (Bunge, 1982). A theoretical texture was used for the investigations. The texture consists of an ideal orientation, $g_{0}=\left\{90^{\circ}, 35^{\circ}, 45^{\circ}\right\}$, with a spread range in the form of a Gaussian distribution of the half-width $\omega_{0}=7.5^{\circ}$. This ideal orientation corresponds to the copper type texture $\{112\}<111>$. The ODF was calculated with $l_{\max }=34$ (Figure 1). The single crystal stiffnesses for copper are displayed in Table 1. Figure 2 presents results for the Voigt, Reuss and Hill values of Young's modulus

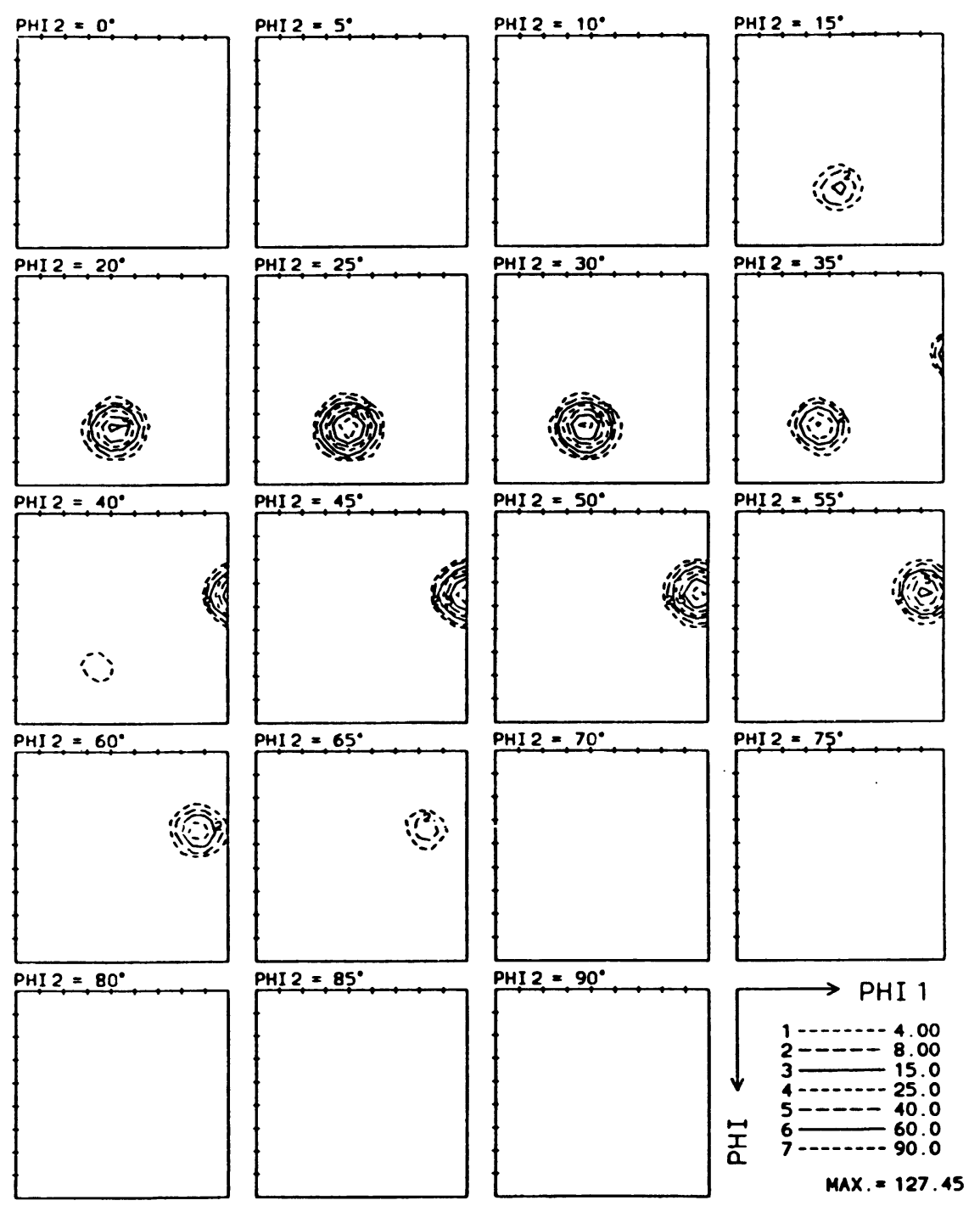

Figure 1 Theoretical texture with an ideal orientation $g=\left\{90^{\circ}, 35^{\circ}, 45^{\circ}\right\}$ and $\omega_{0}=7.5^{\circ}$. 
Table 1 Single crystal values for the stiffnesses $c_{i j}$ of the examined cubic materials in GPa.

\begin{tabular}{ccccc}
\hline & $c_{11}$ & $c_{12}$ & $c_{44}$ & \\
\hline brass $(\mathrm{Cu}-30 w t . \% \mathrm{Zn})$ & 169.05 & 121.93 & 75.50 & (Bradfield, 1962) \\
$\mathrm{Al}$ & 152.1 & 113.9 & 71.9 & (Landolt-Börnstein, 1979) \\
$\mathrm{Fe}$ & 108 & 62 & 28.3 & (Landolt-Börnstein, 1979) \\
\hline
\end{tabular}

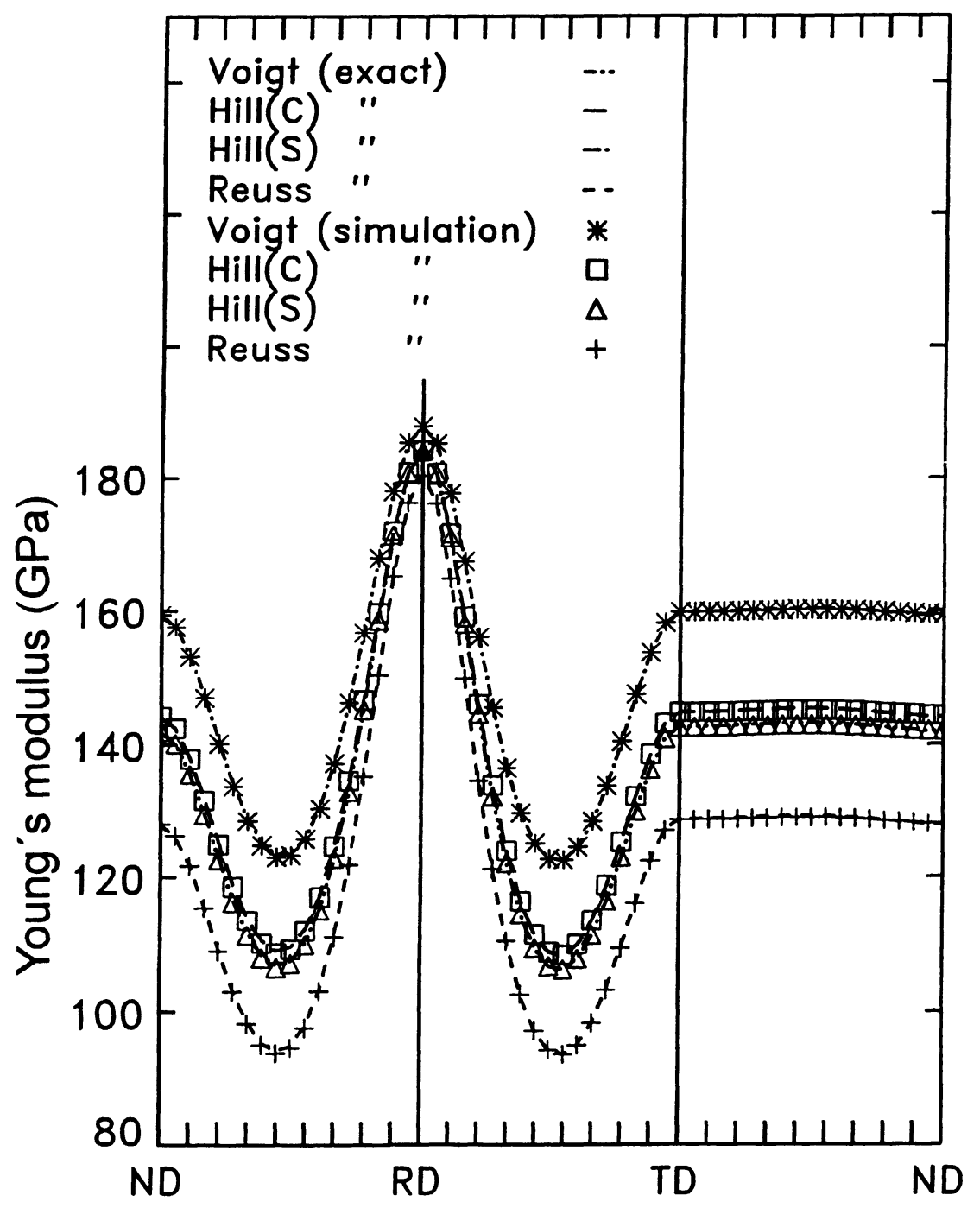

Figure 2 Young's modulus of the copper-type texture of Figure $1\left(l_{\max }=34\right)$. 
E along different high symmetric directions in the sample. The Hill values are defined by:

$$
\begin{gathered}
E_{\text {Hill(C) }} \stackrel{\text { def }}{=} \frac{1}{2}\left(E_{\text {Voigt }}+E_{\text {Reuss }}\right), \\
E_{\text {Hill(S) }} \stackrel{\text { def }}{=}\left(\frac{1}{2}\left(E_{\text {Voigt }}^{-1}+E_{\text {Reuss }}^{-1}\right)\right)^{-1} .
\end{gathered}
$$

The first section of the figure shows the course of Young's modulus between normal (ND) and rolling direction (RD), the second that in the sheet plane and the third depicts that between transverse (TD) and normal direction. The values of the ODF-simulation method and those of the coefficient method which are only affected with errors resulting from the ODF-calculation and therefore are in the sense of ODF-simulation the exact values display a very good agreement. For that reason it is guaranteed that the additional errors for the effective constants caused by the ODF-simulation method are negligible compared with that due to the ODF-calculation.

Testing the convergence of the series expansion method employed for the ODFcalculation is an interesting application of our ODF-simulation scheme. Figure 3 presents the direction dependence of $E_{\text {Hill(S) }}$ for different cut-off values $l_{\max }$ of the series expansion. The material is the same as in Figures 1 and 2. The curve for $l_{\max }=34$ coincides with that of the exact coefficient method, which only requires the coefficients up to $l=4$ (Bunge, 1982) and therefore is not suitable to examine the convergence of the series expansion of the ODF. With decreasing cut-off value the curves become smoother. This nearly ideal orientation shows that an $l_{\max }$ value of at least 22 has to be used to represent strong textures satisfactorily.

\section{Investigations of Experimental Textures}

After carrying out these preliminary investigations we present elastic constants for some substances whose ODF results from pole figure measurements. The single crystal data are displayed in Tables 1, 2 and 3. Figures 4 to 11 show the ODF and the resulting direction-dependence of Young's modulus for these substances computed with the cluster method. The calculations were carried out for a cluster of $7^{3}$ to $8^{3}$ cubical grains whose orientations were determined by the ODF-simulation method. There is no orientation correlation between the grains since the assignment of the orientations to the individual grains is carried out with a random generator. To proof the results we calculated additionally the bounds of Voigt, Reuss and the resulting Hill values.

\section{Experimental Textures of Cubic Materials}

For the ODF-analysis three incomplete pole figures - (110), (200), (211) for bodycentred cubic and (111), (200), (220) for face-centred cubic metals - were measured with Co $K_{\alpha}$-radiation in steps $\Delta \alpha=5^{\circ}, \Delta \beta=3.6^{\circ}$ up to $\alpha_{\max }=70^{\circ}$ using an automatic $\mathrm{X}$-ray texture goniometer. The inversion of the pole figures was carried out using an iterative positivity method of the pole figures. The maximum series expansion degree $l_{\max }$ was 34 . The complete ODF was then obtained by the positivity method of the even ODF (Dahms, 1989).

In $95 \%$ cold-rolled copper a strong texture was developed with a maximum value of $19.75 \times$ random (Figure 4 ). The texture can be characterised by the tube (continuous line) of the orientation distribution function between a brass-type orientation $\left(g=\left\{35^{\circ}\right.\right.$, $\left.45^{\circ}, 0^{\circ}\right\}$ or $\{011\}<211>$ ) and copper type orientation. Young's modulus attains relative 


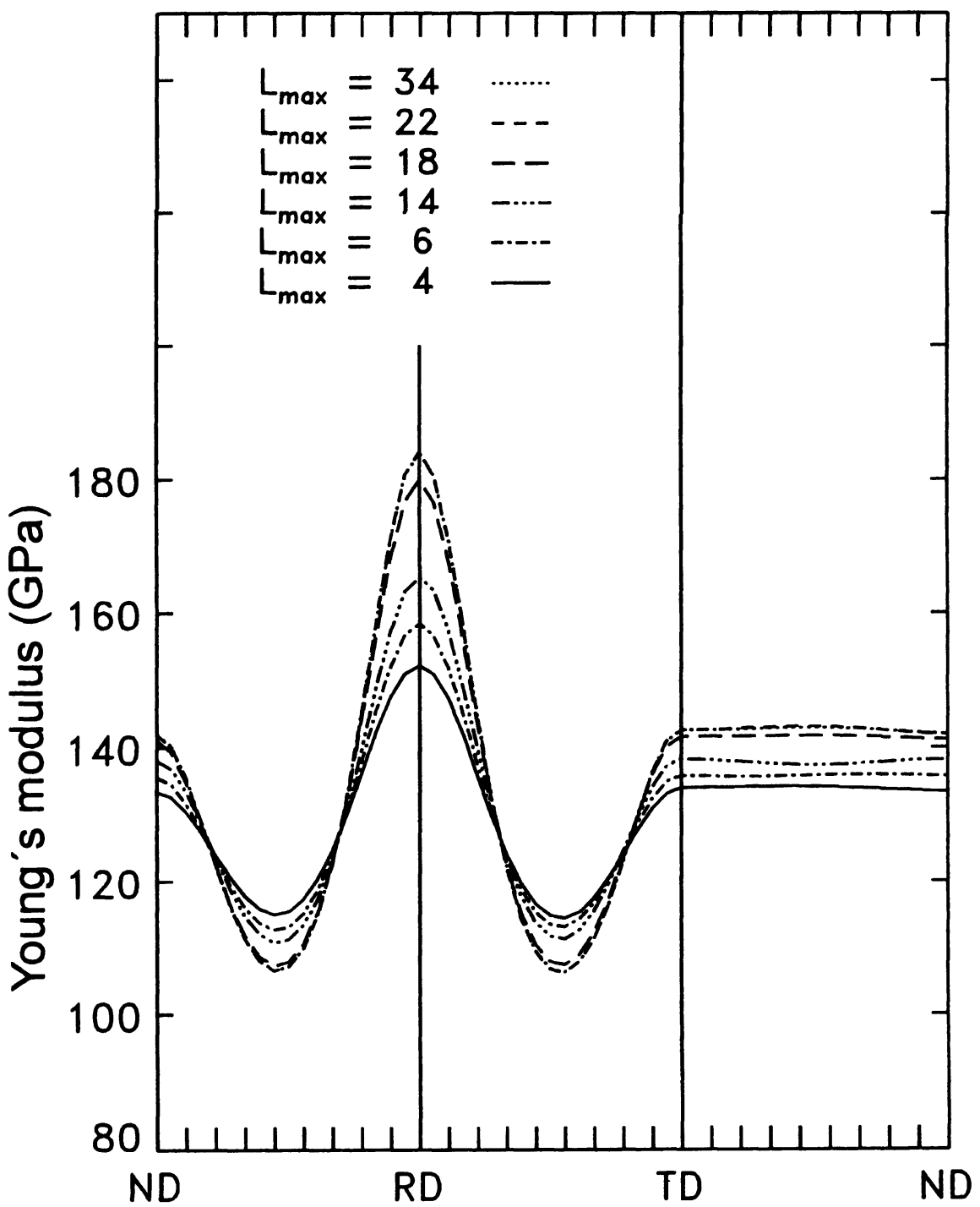

Figure $3 E_{\text {Hill(S) }}$ for different cut-off values $l_{\max }$ of the series expansion method calculated with the ODF-simulation scheme. Note that the exact method to calculate the Hill values need only the series expansion coefficients $C_{l}^{\mu \nu}$ up to $l=4$ (Bunge, 1982).

maxima in rolling, transverse and normal direction which is typical for cold-rolled fccmetals with high relative stacking fault energy. The results of the cluster method confirm the values of Hill.

The texture of the $95 \%$ cold-rolled brass $(\mathrm{Cu}-30 \mathrm{wt} . \% \mathrm{Zn})$ is different from that of the copper. It can be represented by the brass-type orientation only (Figure 5). This is also mirrored in the course of the $E$-modulus: The maximum of $E$ in the sheet is reached in the transverse, the minimum at $45^{\circ}$ to the normal direction. 
Table 2 Single crystal values for the stiffnesses of Ti in GPa.

\begin{tabular}{ccccccc}
\hline & $c_{11}$ & $c_{12}$ & $c_{13}$ & $c_{33}$ & $c_{44}$ & \\
\hline $\mathrm{Ti}$ & 162.92 & 93.43 & 62.0 & 180.28 & 46.59 & (Bradfield, 1962) \\
\hline
\end{tabular}

Table 3 Single crystal values for the stiffnesses of the orthorhombic materials in GPa. The martensitic state of the shape memory alloy $\mathrm{CuZnAl}$ has approximately orthorhombic crystal symmetry. The absolute values for the non-vanishing stiffnesses $c_{15}, c_{25}, c_{35}, c_{46}$ of the monoclinic $\mathrm{CuZnAl}$ are always $\leq 10 \mathrm{GPa}$ and therefore negligible compared with the other non-vanishing stiffnesses.

\begin{tabular}{lcccccccccc}
\hline & $c_{11}$ & $c_{12}$ & $c_{13}$ & $c_{22}$ & $c_{23}$ & $c_{33}$ & $c_{44}$ & $c_{55}$ & $c_{66}$ & \\
\hline $\mathrm{YBa}_{2} \mathrm{Cu}_{3} \mathrm{O}_{7}$ & 223 & 37 & 89 & 244 & 93 & 138 & 61 & 47 & 97 & (Ledbetter and Ming, 1991) \\
$\mathrm{CuZnAl}^{2}$ & 175 & 118 & 40 & 156 & 150 & 235 & 54 & 28 & 48 & (Rodriguez et al., 1993) \\
\hline
\end{tabular}
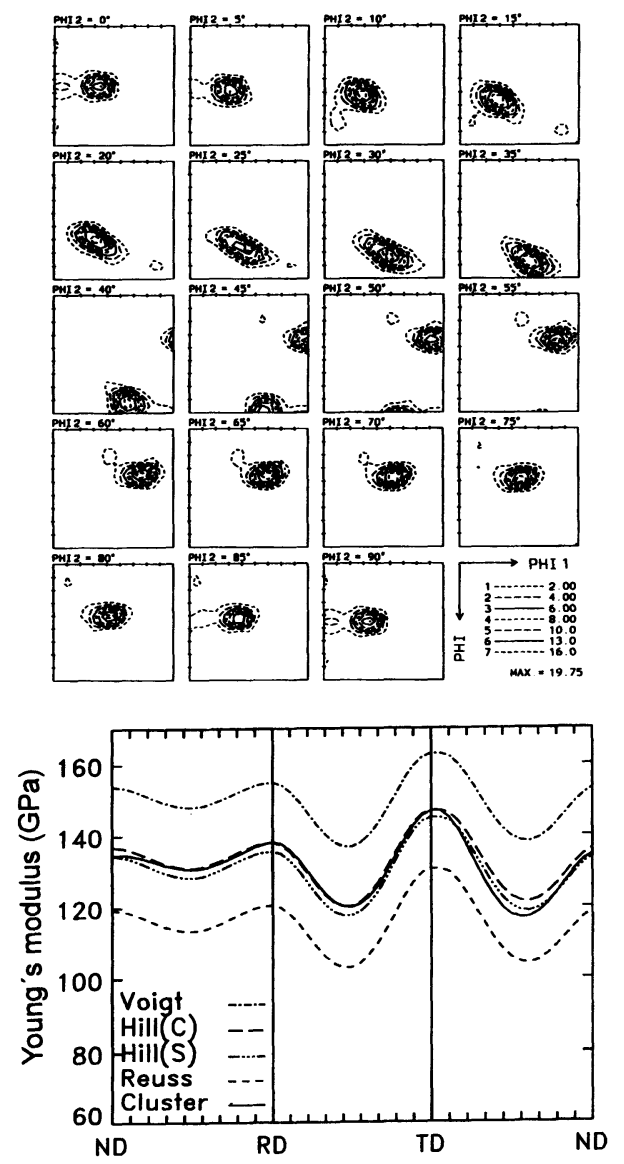

Figure 4 ODF and Young's modulus of a 95\% cold-rolled copper. 

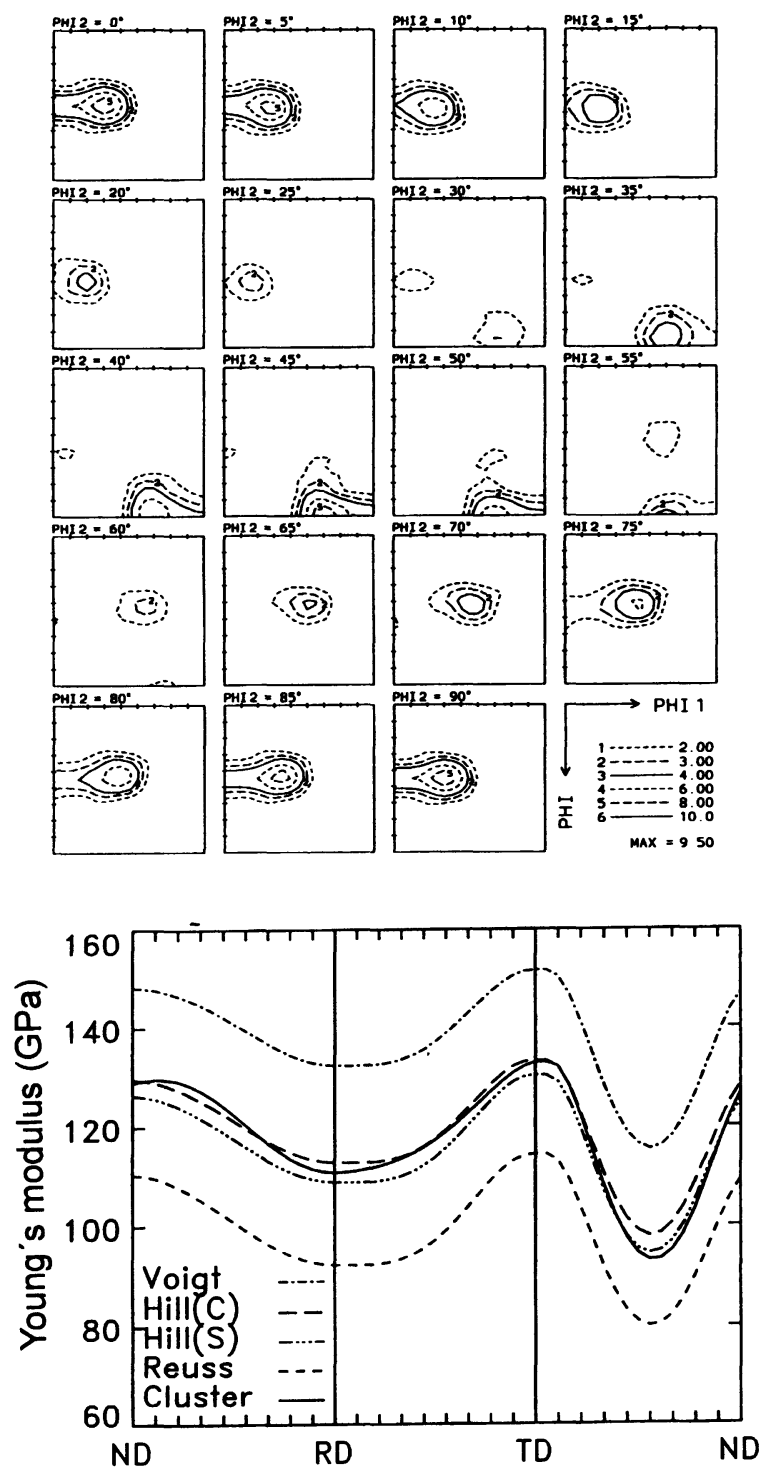

Figure 5 ODF and Young's modulus of a 95\% cold-rolled brass (Cu-30wt,\%Zn).

In a cold-rolled and recrystallised aluminium two ideal orientations were developed (Figure 6) i.e. a stronger cube texture $\left(g=\left\{0^{\circ}, 0^{\circ}, 0^{\circ}\right\}\right.$ or $\left.\{100\}<001>\right)$ with an orientation density of $22.12 \times$ random and a minor $S$-orientation $\left(g=\left\{59^{\circ}, 37^{\circ}, 63^{\circ}\right\}\right.$ or $\{123\}<634>$ ). The direction dependence of Young's modulus is characteristical for recrystallised fcc-metals (Wassermann, 1962). The relative extrema coincide with those of a single crystal in $\{100\}<001>$ orientation. Single crystals of aluminium possess a very low anisotropy (Zeneranisotropy $=1.23$ ). Hence, the difference between the 

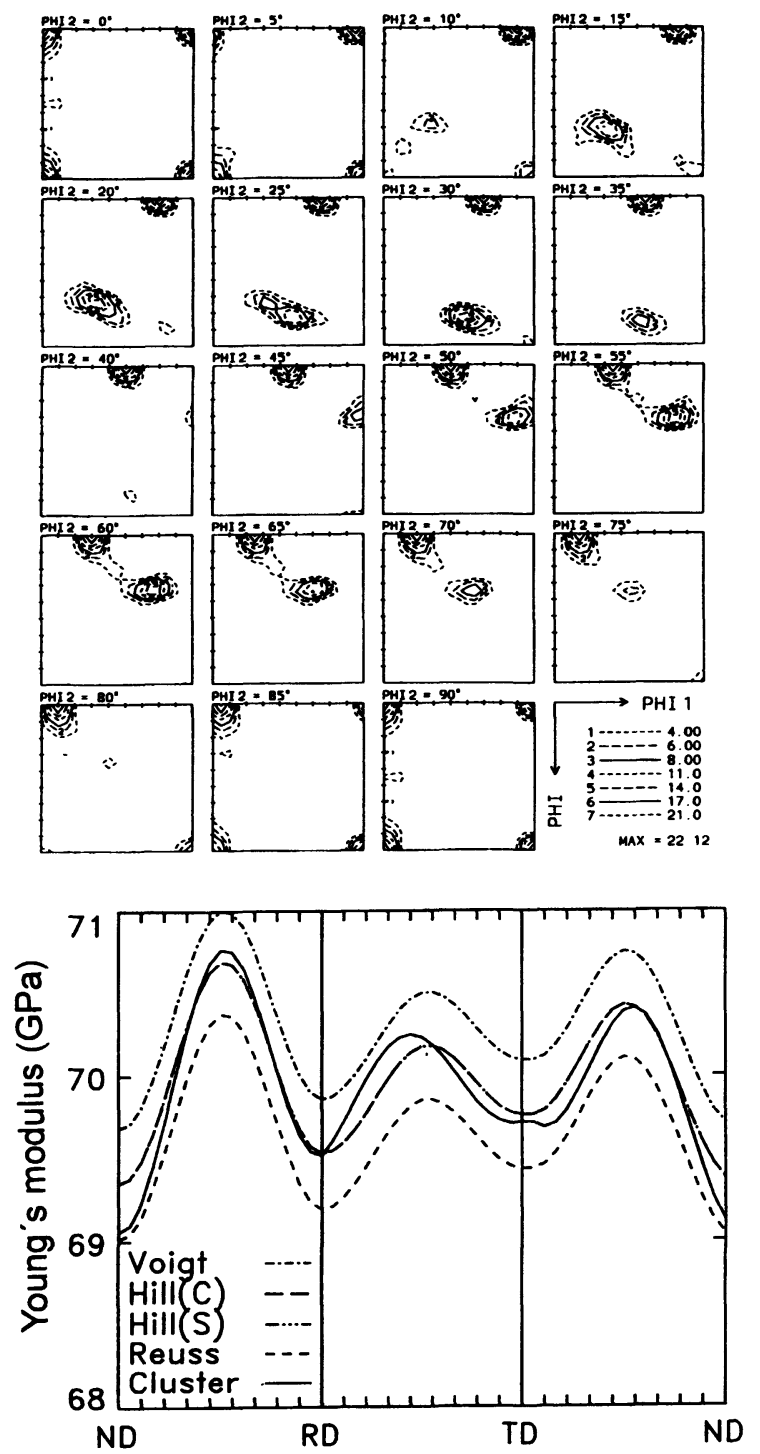

Figure 6 ODF and Young's modulus of a recrystallised aluminium.

bounds of Voigt and Reuss is also very low and the two Hill values are identical within the accuracy of this displaying technique, although the strength of this aluminium texture is higher than that of copper and brass in Figures 4 and 5. The results of the cluster method lie always within these narrow bounds of Voigt and Reuss. Therefore we are in the position to guarantee the reliability of our cluster approach.

ARMCO-iron was cold rolled in two different modes, i.e. $90 \%$ straight-rolled and 93\% cross-rolled (with a rotation of the rolling direction through $90^{\circ}$ after each individual pass). The straight-rolling texture can be described by two tubes of preferred orientations (Figure 7), i.e. by $\langle 110\rangle$ and $<111>$ fibre textures. The $<110>$ fibre is shown in cuts 

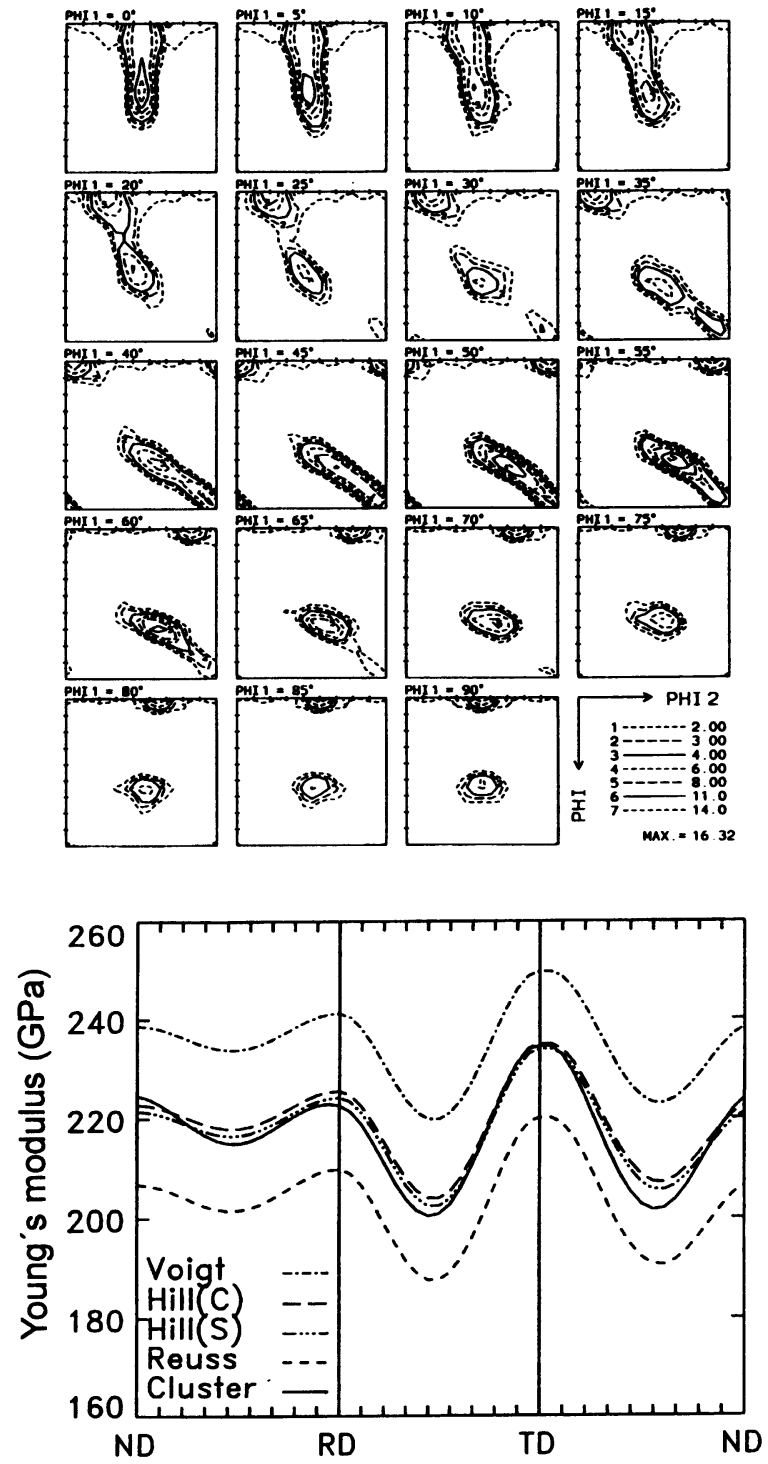

Figure 7 ODF and Young's modulus of a 90\% straight-rolled ARMCO-iron.

$\varphi_{1}=0^{\circ}$ which extends from the orientation $\{001\}<110>\left(\left\{0^{\circ}, 0^{\circ}, 45^{\circ}\right\}\right)$ to $\{112\}<110>$ $\left(\left\{0^{\circ}, 54.7^{\circ}, 45^{\circ}\right\}\right)$. The fibre axis lies parallel to the rolling direction. The second fibre $<111>$ whose axis lies parallel to the normal direction, is seen in all the $\varphi_{1}$ cuts. It runs between $\{112\}<110>$ and $\{111\}<211>$ orientations. The elastic constants calculated with the cluster method are approximately the same as the theoretically unfounded Hill values. In cross-rolled iron a strong $\{001\}<110>$ orientation with an extremely high intensity of $41.62 \times$ random and a week fibre $<111>$ were developed (Figure 8). The course of Young's modulus reveals the typical 4-fold symmetry of cross-rolled materials. 

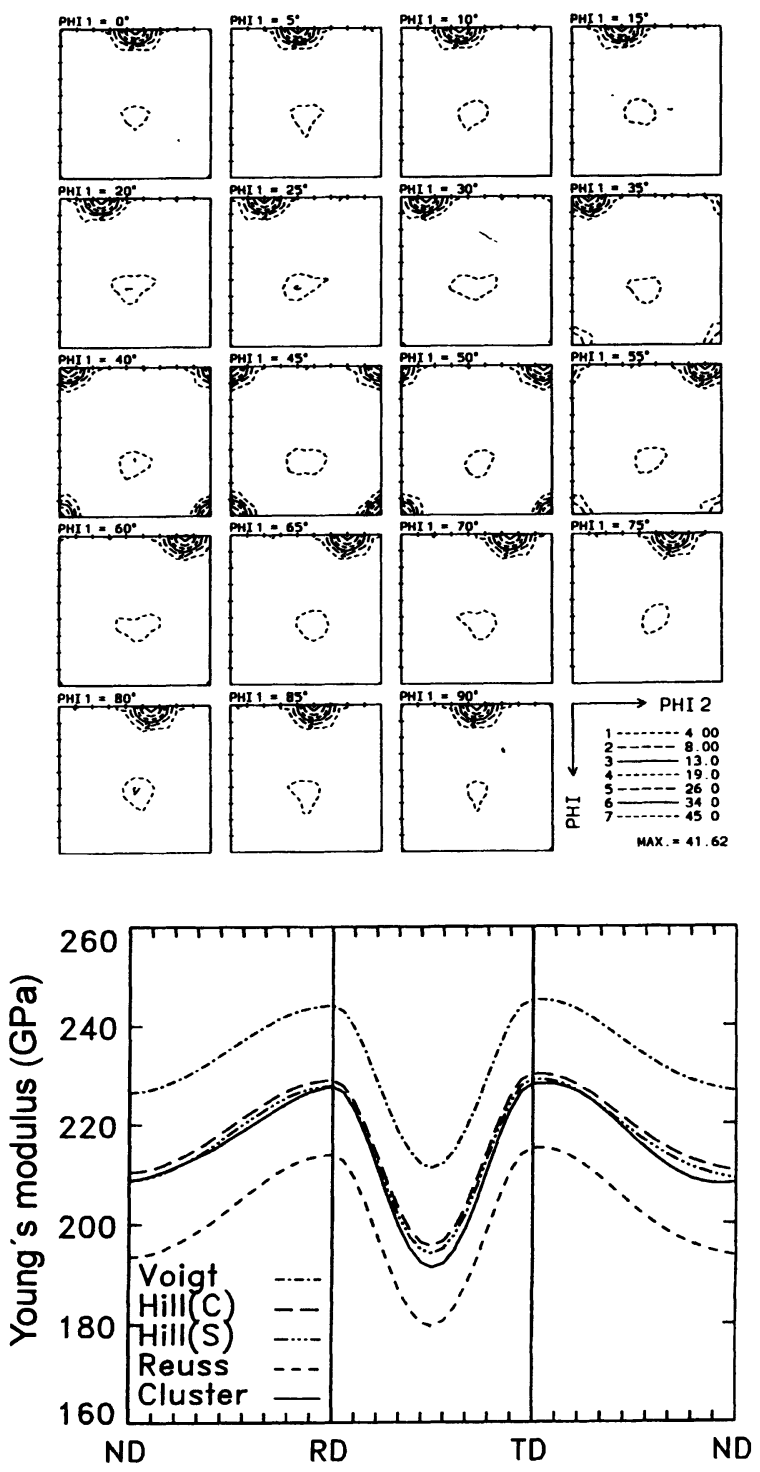

Figure 8 ODF and Young's modulus of a 93\% cross-rolled ARMCO-iron.

\section{Experimental Textures of Low Crystal Symmetry Materials}

For the ODF-analysis of a recrystallised titanium which has hexagonal crystal symmetry five incomplete pole figures, i.e. (01.0), (00.2), (01.1), (01.2), (11.0), were measured. The complete ODF was calculated by the positivity method with the maximum series expansion degree $l_{\max }=23$ (Figure 9). The texture can be represented with two components and a fibre between them. The stronger component is $\{00.1\}<11.0\rangle+40^{\circ}$ TD $\left(\left\{0^{\circ}, 40^{\circ}, 30^{\circ}\right\}\right.$, i.e. the orientation $\{00.1\}<11.0>$ rotated $40^{\circ}$ towards the transverse 

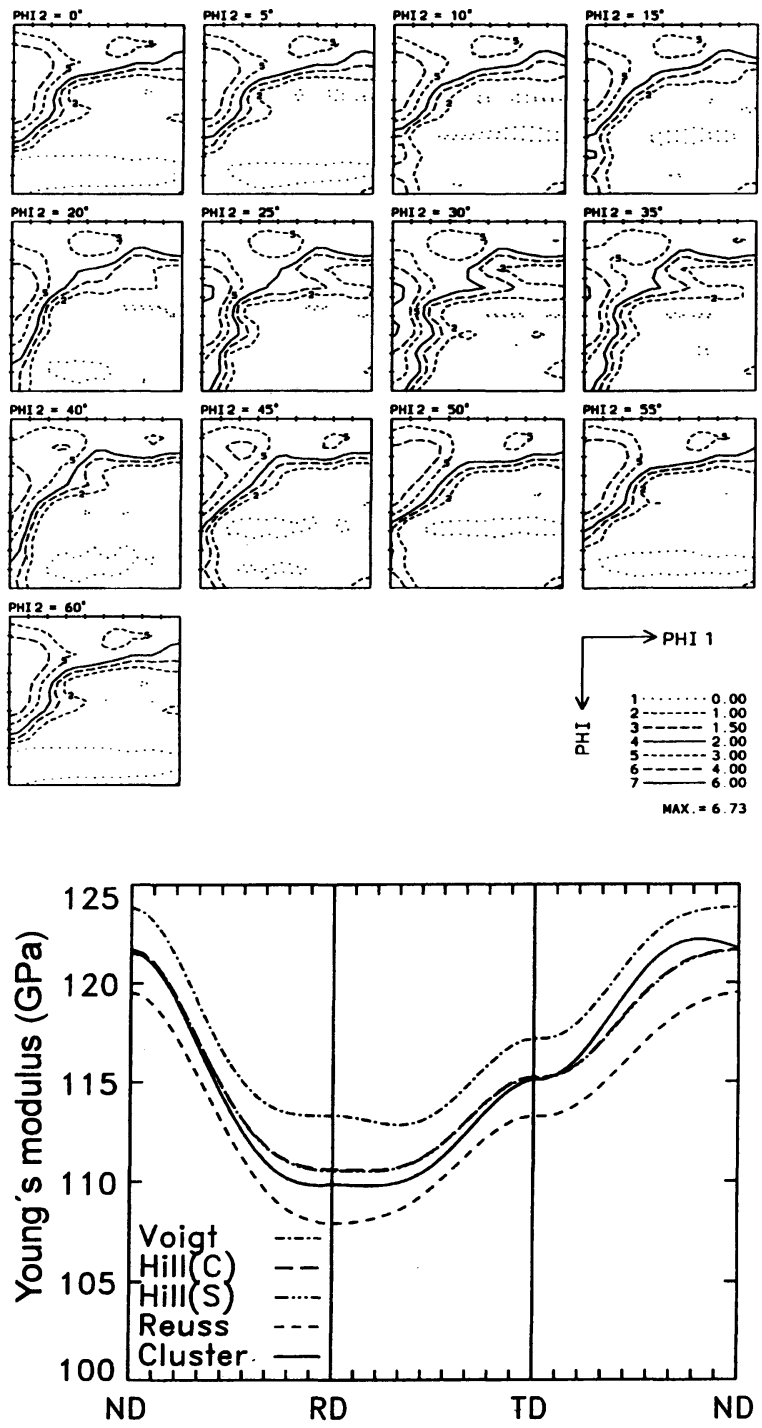

Figure 9 ODF and Young's modulus of a recrystallised titanium.

direction). The other component is $\{00.1\}<10.0>+40^{\circ} \mathrm{TD}\left(\left\{0^{\circ}, 40^{\circ}, 0^{\circ}\right\}\right)$. The curve of Young's modulus reveals the remarkable result that the maximum value of the $E$ modulus which lies in the normal direction is much higher than the values of $E$ in the sheet plane.

The texture in a magnetically aligned $\mathrm{YBa}_{2} \mathrm{Cu}_{3} \mathrm{O}_{7}$ superconducting material was investigated. The ODF was calculated with five incomplete measured pole figures as tetragonal crystal symmetry $\left(l_{\max }=23\right.$ ) (Figure 10). Four pole figures of them are superposed, i.e. $(110)+(103)$, (005) + (104), (113), $(006)+(200)$ and (116) + (123). A strong (001) fibre texture whose axis lies parallel to the magnetic field was developed 

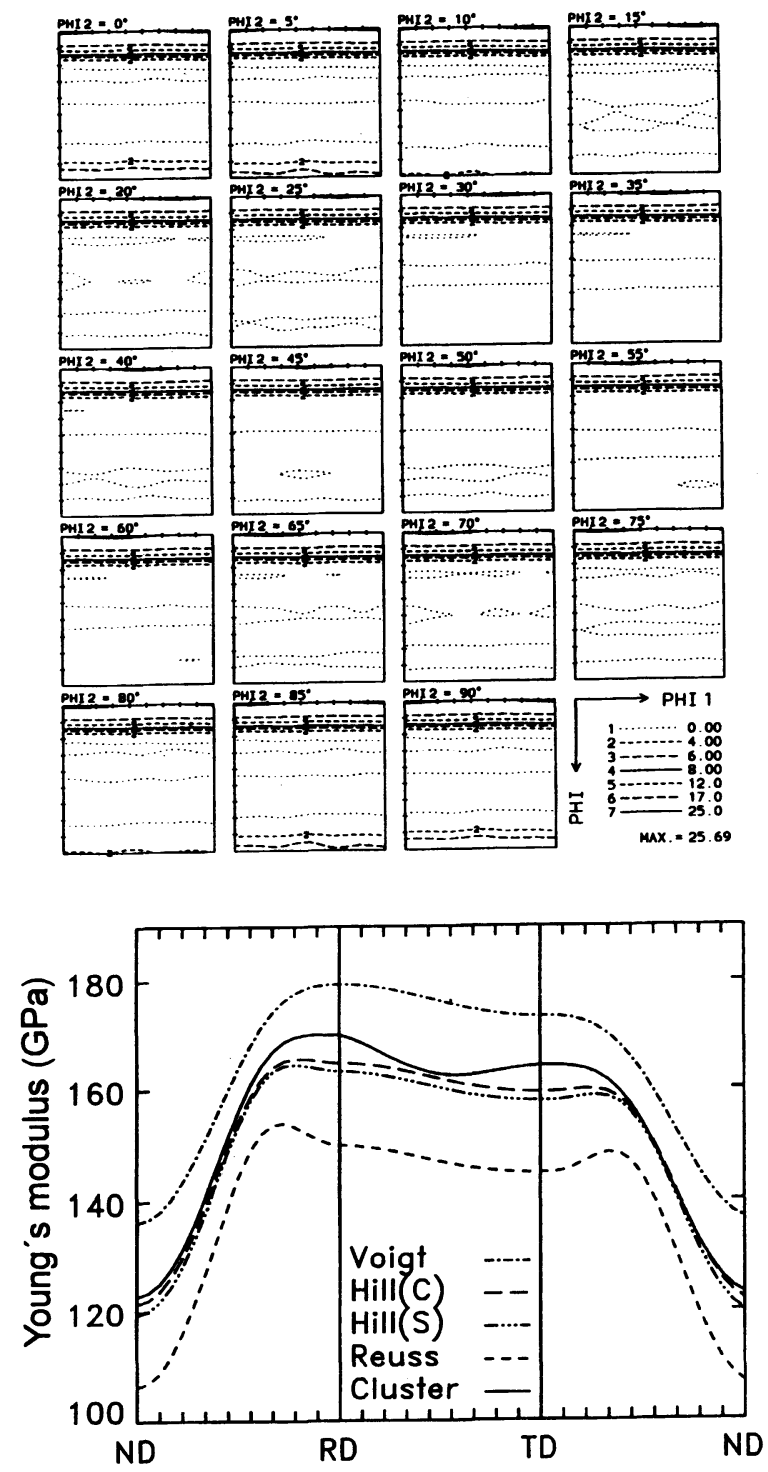

Figure 10 ODF and Young's modulus of a magnetically aligned $\mathrm{YBa}_{2} \mathrm{Cu}_{3} \mathrm{O}_{7}$ superconductor.

(Liu, 1990). The rotational symmetry relative to the fibre axis is mirrored in the curve of Young's modulus: In the sheet plane the modulus is nearly constant while the curve in $\mathrm{RD} / \mathrm{ND}$-direction is very similar to that in TD/ND-direction.

The texture development of the $\mathrm{CuZnAl}$ shape-memory-alloy was studied in the phasetransformed martensitic state after $96 \%$ hot-rolling reduction. This martensitic state has approximately orthorhombic crystal symmetry which leads to decisive simplifications in the ODF-analysis. The complete ODF was calculated with $l_{\max }=23$ using twelve incompletely measured pole figures. Six of them are the result of two overlapping 

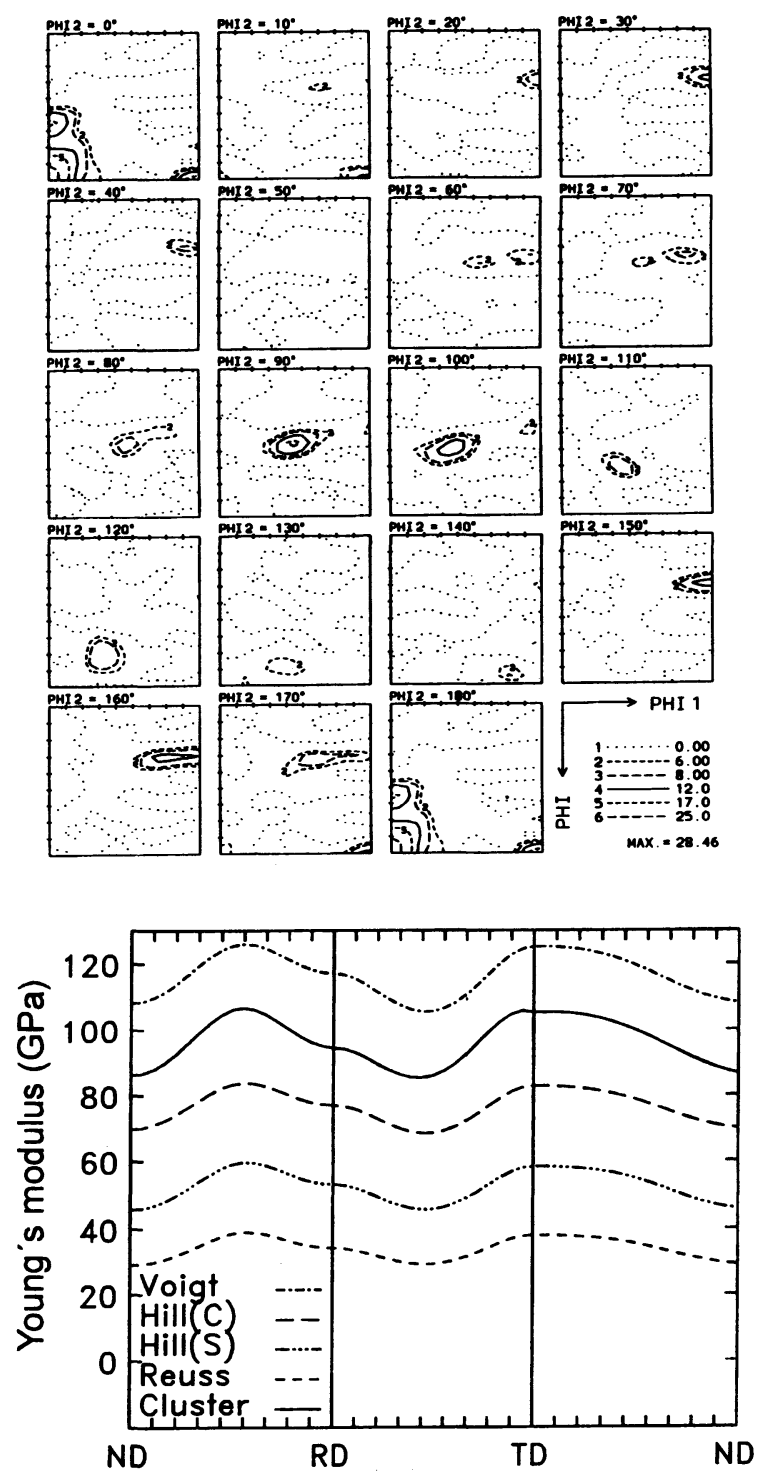

Figure 11 ODF and Young's modulus of a $96 \%$ hot-rolled CuZnAl shape-memory-alloy.

diffraction peaks. The ODF is shown in Figure 11. The texture can be described by six components which must be due to a strong deformation texture in the cubic parent state with subsequent oriented phase transformation to the martensitic state (Park, 1994). In this case there exists an enormous difference between the bounds of Voigt and Reuss since the crystals of this alloy show an extremely high anisotropy. Therefore the two Hill values differ considerably. Hence, the results of the cluster method which show significant deviations from the theoretical non-founded Hill values are the only reliable elastic constants for this material. 


\section{CONCLUSIONS}

Effective elastic constants of textured polycrystalline aggregates were calculated by a method based on the calculation of the displacement field within a cluster of $7^{3}$ or $8^{3}$ grains, taking the boundary conditions at the grain boundaries into account. The method thus provides the exact values of the effective constants for the considered cluster (A series truncation effect due to the finite number of basis functions used for the representation of the displacement field is small and can be neglected). In the particular calculations presented here, an unrealistic grain shape, i.e. cubic grains, was used. This has some influence on the results which will be further studied by using other, more realistic grain shapes.

The calculations were compared with the Voigt and Reuss boundaries as well as with the Hill averages of these. Calculations were made for a variety of textures and for a broad range of elastic anisotropies ranging from near to isotropy as in aluminium to very strong anisotropies in some shape memory alloys.

In the case of smaller anisotropies the present cluster calculations were always near the Hill averages which are close together in this case. In the case of strong elastic anisotropies of the constitutive crystals, e.g. in shape memory materials the Hill averages are far separated and the cluster results are found outside these values. In this case the iterative averaging procedure leading to a unique "generalised Hill average" does not converge towards the effective constants as calculated by the cluster model.

\section{References}

Bradfield, G. (1962). Private communication to Kneer, G. (quoted in (Kneer, (1965)).

Bunge, H. J. (1982). Texture Analysis in Materials Science, Butterworths, London.

Dahms, M. and Bunge, H. J. (1989). The iterative series-expansion method for quantitative texture analysis. A general outline. J. Appl. Cryst., 22, 439.

Hill, R. (1952). The elastic behaviour of a crystalline aggregate. Proc. Phys. Soc., A65, 351.

Kiewel, H. and Fritsche, L. (1994). Calculation of average elastic moduli of polycrystalline materials including $\mathrm{BaTiQ}_{3}$ and high-Tc superconductors. Materials Science Forum, 157-162, 1609.

Kiewel, H. and Fritsche, L. (1994). Calculation of effective elastic moduli of polycrystalline materials including non textured samples and fiber textures. Phys. Rev., B50, 5.

Kneer, G. (1964). Zur Elastizität vielkristalliner Aggregate mit und ohne Textur, Doctoral Thesis, Technische Universität Clausthal.

Kneer, G. (1965). Über die Berechnung der Elastizitätsmoduln vielkristalliner Aggregate mit Textur. Phys. Stat. Sol., 9, 825.

Kröner, E. (1958). Berechnung der elastischen Konstanten des Vielkristalls aus den Konstanten der Einkristalle. Z. Phys., 151, 504.

Kröner, E. (1977). Bounds for effective elastic moduli of disordered materials. J. Mech. Phys. Solids, 25, 137.

Kumar, S. (1992). Computer simulation of 3D materials microstructure and its application in the determination of mechanical behavior of polycrystalline materials and engineering structures, $\mathrm{Ph}$. D. Thesis, Pennsylvania State University.

Landolt-Börnstein (1979). Elastic, Piezoelectric and Related Constants, Group III of New Series Vol. 11, Springer, Heidelberg.

Ledbetter, $\mathrm{H}$. and Ming, L. (1991). Monocrystal elastic constants of orthotropic $\mathrm{Y}_{1} \mathrm{Ba}_{2} \mathrm{Cu}_{3} \mathrm{O}_{7}: \mathrm{An}$ estimate. J. Mater. Res., 6, 2253.

Liu, W. P., Park, N. J. and Bunge, H. J. (1990). Textures in ceramic superconductors. Textures and Microstructures, 13, 41.

Morawiec, A. (1994). Review of deterministic methods of calculation of polycrystal elastic constants. Textures and Microstructures, 22, 139.

Morris, P. R. (1971). Iterative scheme for calculating polycrystal elastic constants. Int. J. Eng. Sci., 9, 917.

Park, N. J. and Bunge, H. J. (1994). Texture transformation due to martensitic phase transformation in CuZnAl shape memory alloys. Materials Science Forum, 157-162, 563. 
Reuss, A. (1929). Berechnung der Flie $\beta$ grenze von Mischkristallen auf Grund der Plastizitätsbedingung für Einkristalle. Z. Angew. Math. Mech., 9, 49.

Rodriguez, P. L., Lovey, F. C., Guenin, G., Pelegrina, J. L., Sade, M. and Morin, M. (1993). Elastic constants of the monoclinic 18R martensite of a Cu-Zn-Al alloy. Acta Metall. Mater., 41, 3307. Voigt, W. (1910). Lehrbuch der Kristallphysik, Teubner, Berlin.

Wassermann, G. and Grewen, J. (1962). Texturen metallischer Werkstoffe, Springer, Heidelberg. 